\title{
Structural Studies of Toll-like Receptor 7
}

Zhikuan Zhang, Graduate School of Pharmaceutical Sciences, The University of Tokyo, Tokyo, Japan Umeharu Ohto, Graduate School of Pharmaceutical Sciences, The University of Tokyo, Tokyo, Japan Toshiyuki Shimizu, Graduate School of Pharmaceutical Sciences, The University of Tokyo, Tokyo, Japan

Toll-like receptor 7 (TLR7) is one of many nucleic acid receptors in the innate immune system. It is considered as a viral single-stranded RNA (ssRNA) receptor, although it can also be activated by endogenous or artificially-dosed RNAs and several natural or synthetic small ligands such as guanosine and imidazoquinoline derivatives. Thus this receptor protein is closely related to viral infectious diseases and autoimmune diseases and its agonistic or antagonistic ligands have a great application as immune modulators. However, the atomic structure and ligand-recognition and activation mechanism of TLR7 were largely unknown. We first determined the crystal structures of TLR7 in small ligand- and ssRNA-bound activated forms (Zhang et al., Immunity., 2016). Through structural analysis we elucidated that TLR7 was a dual receptor for guanosine nucleoside and nonterminal uridine containing ssRNAs. Under natural condition, the activation of TLR7 would be induced by guanosine in an ssRNA-dependent manner. On the other hand, high-affinity small ligands would activate TLR7 via the guanosine-binding site in an ssRNA-independent manner. 